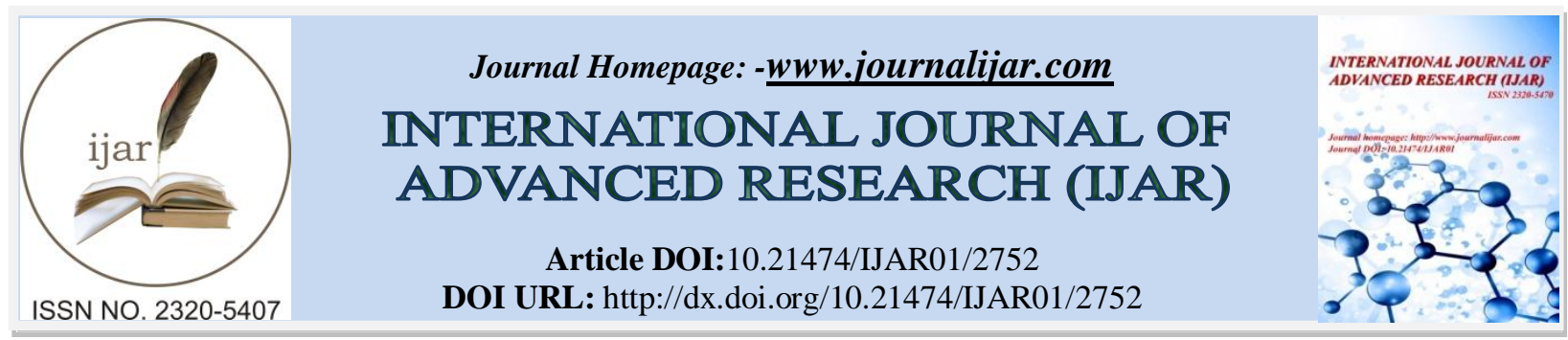

RESEARCH ARTICLE

\title{
CHECKLIST OF FLOWERING PLANTS SURROUNDING THE WETLANDS IN VADODARA DISTRICT.
}

Mamta K. Joshi ${ }^{1}$,Rupesh Maurya ${ }^{1}$, Umerfaruq Qureshi ${ }^{1}$ and Hitesh A. Soalnki ${ }^{2}$.

1. Research Scholar, Department of Botany, University School of Sciences, Gujarat University, Ahmedabad-380 009.

2. Professor, Department of Botany, University School of Sciences, Gujarat University, Ahmedabad-380 009.

\section{Manuscript Info}

Manuscript History

Received: 15 November 2016

Final Accepted: 17 December 2016

Published: January 2017

\section{Abstract}

Wetland is the most productive ecosystems in the world. Aquatic plants are vital components for the proper management of wetland ecosystem for biological productivity and support various organisms. In the present study, diversity of flowering plants surrounding the wetlands in Vadodara District was carried out at selected 19 wetlands in 6 talukas.

Copy Right, IJAR, 2016,. All rights reserved.

\section{Introduction:-}

Wetlands are "lands transitional between terrestrial and aquatic systems where the water table is usually at or near the surface or the land is covered by shallow water." Its features were (a) at least intermittently, the land supports chiefly hydrophytes (b) the substrate is chiefly undrained hydric soil (c) the substrate is non-soil and is saturated with water at some time throughout the growing season of the year.

Over the last era significant struggle which directed to the conservation and wise use of wetlands (Jonauskas, 1996; Finlayson, Hall and Bayliss 1998; Blackman, 1995). However, available knowledge or information for some wetlands are at the most basic level, as information on physical and ecological features, values and benefits, land tenure and uses, threats and disturbances, and monitoring and restoration of all wetlands is not available (Finlayson, Hall and Bayliss 1998). The wise use and conservation of wetlands will be partly needful on a greatly expanded information base. Data on the ecological character of wetlands, which is the amount of wetland loss and degradation, conservation measures and the success of monitoring strategies will be required. Moreover, this information base requires linkage and integration with executive processes (Finlayson, 1995). Classification and account of wetlands are processes designed to deliver a key point on wetlands and their resources (Finlayson, 1933). Aquatic biodiversity is dependent on hydrologic regime; geological conditions and efforts are being made to conserve the biodiversity found in wetlands, streams and rivers. The goal of this irreplaceable biodiversity is to minimize its loss through sustainable management and conservation practices. The first step in conservation of biodiversity is to assess the diversity of natural resources present and identify those, which are important and most irreplaceable (Groombridge \& Jenkins 1998). The total numbers of aquatic plant species exceed 1200 and a partial list of animal for aquatic and wetland system is given by Gopal (1995). Wetlands are also important as resting sites for migratory birds. Avifauna species found in India have been listed by Gopal (1995).

Wetlands are one of the most valuable and important natural environments. They provide suitable habitats to innumerable organisms including birds.Wetlands in India cover an area of 58.2 million hectares (Prasad et al., 2002). Of 1340 bird species found in India (Ali and Ripley, 1987), 310 species are known to be wetland birds (Kumar et al., 2005). Mitsch and Gosselink (2000) stated that wetlands help in maintaining biodiversity of flora and 
fauna and it was further emphasized in the study that countless species of birds, mammals, reptiles, amphibians, fish and invertebrate species depend on water and wetland vegetation for their survival. Similar observations on wetlands were also made by Buckton (2007).

\section{Materials and Methods:-}

Study Area: Vadodara is situated on the banks of the river Vishwamitri (whose name is derived from the great saint Rishi Vishwamitra). The city was once called Chandravati, after its ruler Raja Chandan, then Viravati, the abode of the brave, and then Vadpatra because of the abundance of banyan trees on the banks of the Vishwamitri. From Vadpatra it derived its present name Baroda or Vadodara. It is located at $22.30^{\circ} \mathrm{N} 73.19^{\circ} \mathrm{E}$ in western India at an elevation of 39 meters (123 feet) Occupying an area of 4138 sq. km.

In the present study 6 talukas are undertaken which are Vadodara, Karjan, Padra, Savli, Vaghodiya and Dabhoi.

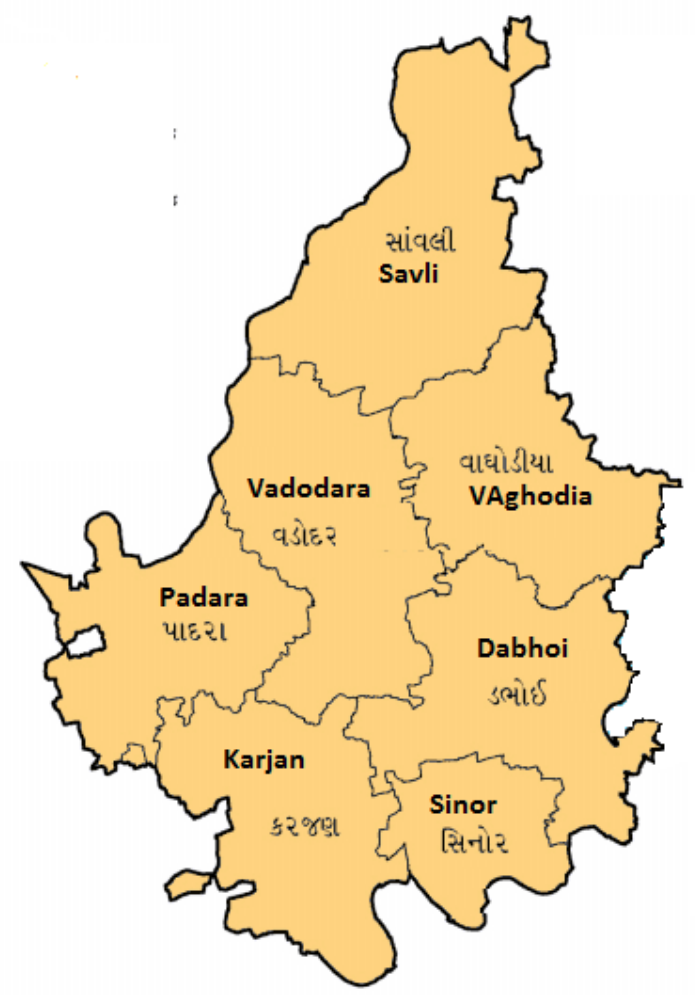

Figure No 1: Map showing talukas of Vadodara District

Methodology: The study has been conducted in wetlands of different talukas of Vadodara district during 2012-14.

Flora: During the field study, plants were documented and plant specimens were also collected along with their flowering and fruiting parts for preparing herbarium. These herbaria are useful for identifying the specimens from the flora. The herbarium sheets were labelled, numbered and deposited in the Herbarium of Gujarat University. The plant species were identified with the help of available literature in the library (Department of Botany, Gujarat University, Ahmedabad). Photographs of plants were captured with a SLR camera.

Fauna: Observations were made by conducting field visits at regular intervals. Fieldwork was conducted from 2012 - 2014. Field visits were made every month during the study period, to monitor three distinct seasons (i.e. winter, summer and monsoon). The observations were carried out with a pair of binoculars and the species were identified using recognized field guides like those of Ali \& Ripley (1983), Grimmett et al. (1998), Rasmussen \& Anderton (2005), etc. 


\section{Result and Discussion:-}

In the present study 420 species and 330 genera of vascular plants belonging to 91 families were reported. Out of 420 species, there are 211 herbs, 94 trees, 81 climbers and 34 shrubs reported (Graph No. 1). List of plants found in the present study were listed in Table No. 1 along with Botanical name, family and local name. In the present study Fabaceae is found largest family (46 species), followed by Poaceae (29 speceae), Asteraceae (23species), Convolvulaceae (20 species) and rest are below 20 species.

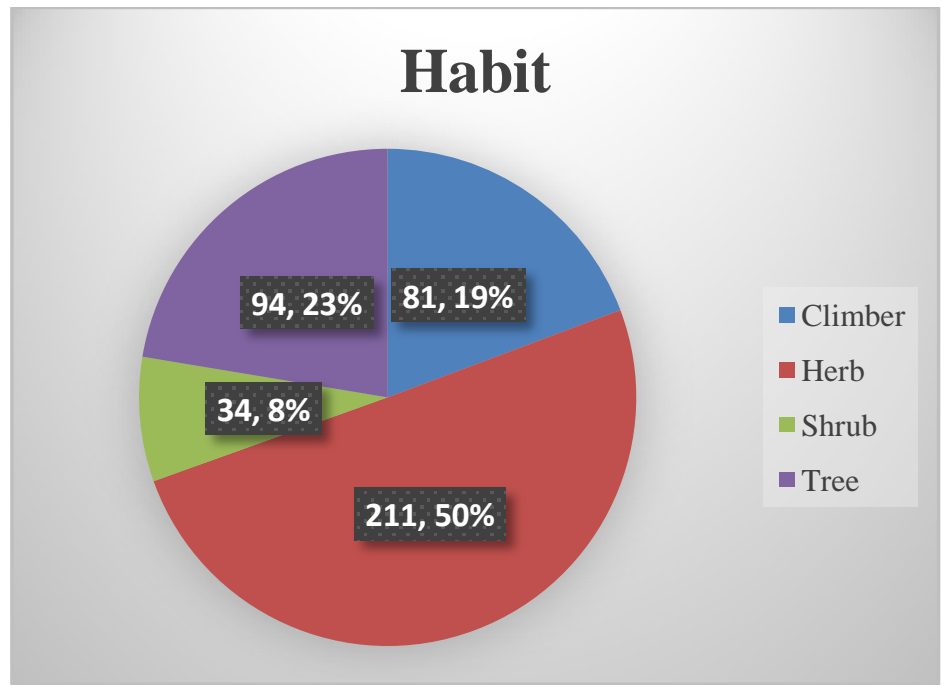

Graph No. 1: Graph showing the habit of flowering plants.

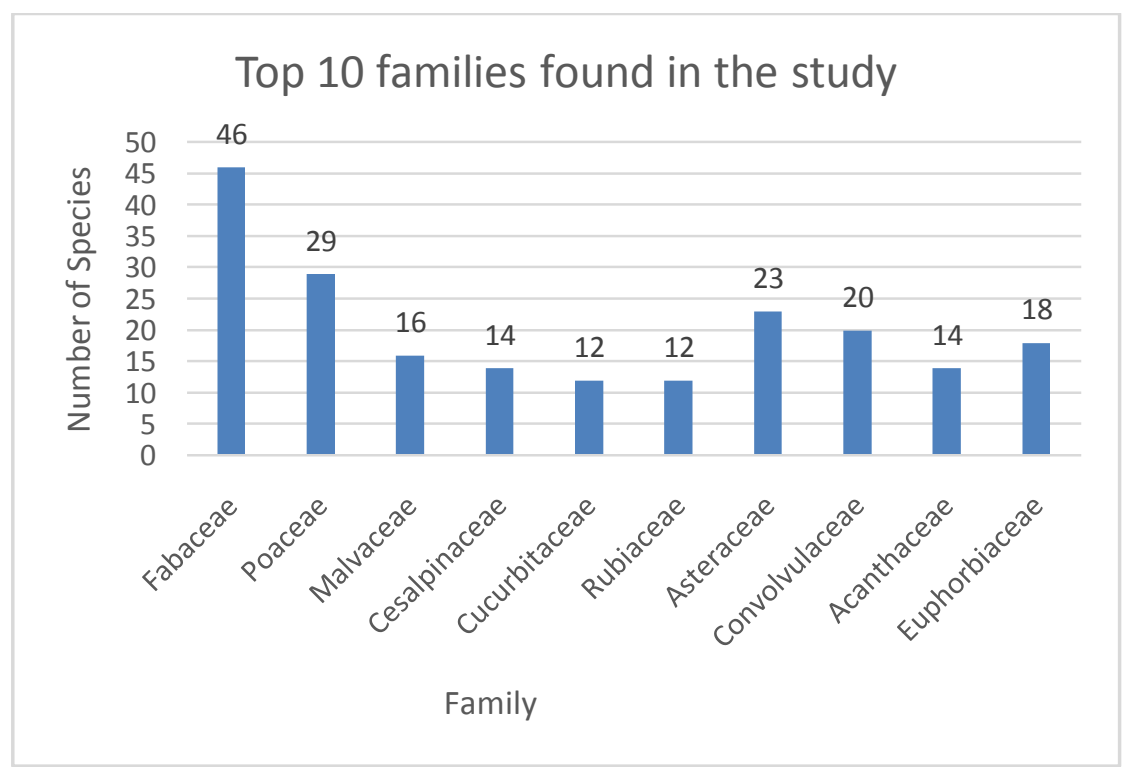

Graph No. 2: Graph showing the top 10 families found in the present study. 
Table No. 1: List of flowering plants found nearby the wetlands in Vadodara district.

\begin{tabular}{|c|c|c|c|}
\hline \multicolumn{4}{|c|}{ List of vascular plants surrouding the Wetlands in Vadodara district. } \\
\hline Sr. No. & Family & Botanical Name & Comman name \\
\hline 1 & \multirow[t]{5}{*}{ Menispermaceae } & Cocculushirsutus (L.) Theob. & Vevdi \\
\hline 2 & & Cocculuspendulus (Forst.) Diels & Orad, Valur \\
\hline 3 & & Tinosporaglabra (Burm . f.) Merril & $\begin{array}{l}\text { Gulvel, Gadu, } \\
\text { Gudaj vel }\end{array}$ \\
\hline 4 & & Nymphaeanauchali Burm. f. & $\begin{array}{l}\text { Kamal,Poyana, } \\
\text { Kamalful }\end{array}$ \\
\hline 5 & & Nymphaeapubescens Willd. & $\begin{array}{l}\text { Kamal,Poyana, } \\
\text { Kamalful }\end{array}$ \\
\hline 6 & Nelumbonaceae & Nelumbonucifera Gaertn. & $\begin{array}{l}\text { Vado kamalful, } \\
\text { Suryakamal }\end{array}$ \\
\hline 7 & Papaveraceae & Argemonemexicana L. & Darudi \\
\hline 8 & \multirow[t]{2}{*}{ Cleomaceae } & Cleome rutidosperma DC. & \\
\hline 9 & & Cleomeviscosa L. & Pili -Tilvan \\
\hline 10 & \multirow[t]{2}{*}{ Cochlosprmaceae } & Cochlospermumreligiosum (L.) Alst. & \\
\hline 11 & & Carseariagraveolens Dalz. & Tandol \\
\hline 12 & Flacourtiaceae & Flacourtiaindica (Burm. f.)Merr. & Kirambira Kirmira \\
\hline 13 & \multirow[t]{4}{*}{ Portulacaceae } & Portulacagrandiflora Hk. f. & Chini-Gulab \\
\hline 14 & & Portulacagranulato-stellulata (Poelln.) Ricceri \& Arrigoni & \\
\hline 15 & & Portulacaoleracea L. & $\begin{array}{l}\text { Motiluni, Lakha } \\
\text { luni }\end{array}$ \\
\hline 16 & & Talinum portulacifolium (Forssk.) Asch.ex Schweinf. & \\
\hline 17 & \multirow[t]{16}{*}{ Malvaceae } & Abelmoschusesculentus (L.) Moench. & Bhinda, bhindi \\
\hline 18 & & Abutilonglaucum (Cav.) Sw. & \\
\hline 19 & & Abutilonindicum (L.) Sw. & Khapat, Dabaliar \\
\hline 20 & & $\begin{array}{l}\text { GossypiumherbaceumL .var. acerifolium (Guill. \& Perr.) } \\
\text { Chevalier }\end{array}$ & Kapas, Desi Kapas \\
\hline 21 & & Hibiscuscannabinus L. & Ambadi \\
\hline 22 & & Hisbisuslobatus (Murr.) O. Ktze. & Tali \\
\hline 23 & & Hibiscusrosa-sinesis. L. & Jasund, Jasvanti \\
\hline 24 & & Hibiscussabdariffa L. & $\begin{array}{l}\text { Khati Bhindi, Lal- } \\
\text { Ambadi }\end{array}$ \\
\hline 25 & & Hibiscusschizopetalous (Masters) Hk . f. & \\
\hline 26 & & Malachracapitata (L.) L. & Pardeshi Bhindo \\
\hline 27 & & Malvastrumcoromandelianum (L.) Garcke & \\
\hline 28 & & Pavoniaodorata Willd. & $\begin{array}{l}\text { Kalavala, Sugandh } \\
\text { Bala }\end{array}$ \\
\hline 29 & & Sidaacuta Burm.f. & Bala \\
\hline 30 & & Sidacordata (Burm.f.) Boiss & Bhoyabala \\
\hline 31 & & Sidacordifolia L. & $\begin{array}{l}\text { Bala, Baladana, } \\
\text { Kharenti }\end{array}$ \\
\hline 32 & & Urenalobata L. & Vagadau Bhindo \\
\hline 33 & \multirow[t]{4}{*}{ Bombacaceae } & Salmaliarubra (Buch.-Ham.) S. Dutta \& P. Harvey & Savar, Shimlo \\
\hline 34 & & Helicteresisora L. & $\begin{array}{l}\text { Maradsing, ati, } \\
\text { Atai }\end{array}$ \\
\hline 35 & & Melochiacorchorifolia L. & Chuncha,Khapat \\
\hline 36 & & Sterculiaurens Roxb. & $\begin{array}{l}\text { Kadai, Kadio, } \\
\text { Kadaya }\end{array}$ \\
\hline 37 & \multirow[t]{3}{*}{ Tiliaceae } & Corchorusaestuans L. & $\begin{array}{l}\text { Chunch, Chhadhari } \\
\text { chunch }\end{array}$ \\
\hline 38 & & Corchoruscapsularis L. & Bor Chhunchi \\
\hline 39 & & Corchorusolitorins L. & \\
\hline
\end{tabular}




\begin{tabular}{|c|c|c|c|}
\hline 40 & & Corchorustridens L. & \\
\hline 41 & & Grewiatiliaefolia vahl. & Dhaman \\
\hline 42 & & Microcospaniculata L. & \\
\hline 43 & & Triumfettapentandra A. Rich. & \\
\hline 44 & & Triunfettarhomboidea Jacq. & \\
\hline 45 & Malpighiaceae & Hiptagebenghalensis (L.) Kurz. & \\
\hline 46 & Zygophyllaceae & Tribulusterrestris L. & Gokharu \\
\hline 47 & \multirow[t]{2}{*}{ Oxalidaceae } & Biophytumsensitivum (L.) DC. & \\
\hline 48 & & Oxaliscorniculata L. & \\
\hline 49 & \multirow[t]{2}{*}{ Balsaminaceae } & Impatiens balsamina L. & \\
\hline 50 & & Impatiensminor (D.C.) Bennet. & \\
\hline 51 & \multirow[t]{2}{*}{ Rutaceae } & Aeglemarmelos (L.) Correa & Bil \\
\hline 52 & & Citrus limon (L.) Osbeck & Limbu \\
\hline 53 & Balanitaceae & Balanitesaegyptiaca (L.) Del. & Ingoriyo \\
\hline 54 & \multirow[t]{2}{*}{ Burseraceae } & Boswelliaserrata Roxb. ex Colebr. & Salai gugal \\
\hline 55 & & Garugapinnata Roxb. & Kakad \\
\hline 56 & \multirow[t]{2}{*}{ Meliaceae } & Azadirachtaindica Juss. & Limbado \\
\hline 57 & & Meliaazedarach L. & Bakam limdo \\
\hline 58 & \multirow[t]{2}{*}{ Celastraceae } & Cassineglauca (Rottb.) O. Ktze. & Bhutzad \\
\hline 59 & & Celastruspaniculatus Willd. & \\
\hline 60 & \multirow[t]{4}{*}{ Rhamnaceae } & Ventilagodenticulata Willd. & \\
\hline 61 & & Ziziphusmauritiana Lamk. & Bor \\
\hline 62 & & Zizyphusoenoplia (L.) Mill. & \\
\hline 63 & & Zizyphusrugosa Lamk. & Ghatbor \\
\hline 64 & \multirow[t]{3}{*}{ Vitaceae } & Cayratiatrifolia (L.) Domin. & \\
\hline 65 & & Cissusquadrangularis L. & Had-sakal \\
\hline 66 & & Cissusrepanda Vahl & \\
\hline 67 & \multirow[t]{3}{*}{ Sapindaceae } & Cardiospermumhalicacabum L. & \\
\hline 68 & & $\underline{\text { Sapindusemarginatus Vahl }}$ & Aritha \\
\hline 69 & & $\underline{\text { Sapinduslaurifolius Vahl. }}$ & Aritha \\
\hline 70 & Anacardiaceae & Anacardiumoccidentale L. & Kaju \\
\hline 71 & \multirow[t]{4}{*}{ Asteraceae } & Lanneacoromandelica (Houtt.) Merr. & Modal \\
\hline 72 & & Mangiferaindica L. & Ambo \\
\hline 73 & & $\underline{\text { Semecarpusanacardium L.f. }}$ & \\
\hline 74 & & Spondiaspinnata (L.f.) Kurz. & Ambado \\
\hline 75 & Moringaceae & Moringaoleifera Lamk. & Sargavo \\
\hline 76 & \multirow[t]{17}{*}{ Fabaceae } & Abrusprecatorius L. & Chanothi \\
\hline 77 & & Aeschynomeneindica L. & \\
\hline 78 & & Aeschynomene Americana & \\
\hline 79 & & Alysicarpusheyneanus Wt. \& Arn. & \\
\hline 80 & & Alysicarpuslongifolius (Rott.ex Spr.) Wt. \& Arn. & \\
\hline 81 & & Alysicarpusvaginalis (L.) DC. & \\
\hline 82 & & Arachishypogea L. & Magfali \\
\hline 83 & & Cajanusscarabaoides (L.) Thouars. & \\
\hline 84 & & Cajanuscajan (L.) Huth & Tuver \\
\hline 85 & & Buteamonosperma (Lamk.) Taub. & Kesudo \\
\hline 86 & & Canavaliagladiata (Jacq.) DC. & \\
\hline 87 & & Canavaliacathartica Thouars & \\
\hline 88 & & Cicerarietinum L. & Channa \\
\hline 89 & & Clitoriaannua Graham & \\
\hline 90 & & Clitoriaternatea $\mathrm{L}$. & \\
\hline 91 & & Crotalariaalbida Heyne ex. Roth & \\
\hline 92 & & $\begin{array}{l}\text { Crotalariafilipes Bth. var. trichophora (Bth. ex Baker) } \\
\text { Cooke }\end{array}$ & \\
\hline
\end{tabular}




\begin{tabular}{|c|c|c|c|}
\hline 93 & & Crotalariajuncea L. & \\
\hline 94 & & Crotalaria medicaginea Lamk. & \\
\hline 95 & & Crotalariaprostrata Rottl. & \\
\hline 96 & & Crotalariatriquetra Dalz. & \\
\hline 97 & & Dalbergialatifolia Roxb. & Sisam \\
\hline 98 & & Dalbergiasissoo Roxb. ex DC. & Sisoo \\
\hline 99 & & Dalbergiavolubilis Roxb. & Patarali \\
\hline 100 & & Derrisscandens (Roxb.) Bth. & \\
\hline 101 & & Desmodiumgangeticum (L.) DC. & \\
\hline 102 & & Erythrinasuberosa Roxb. & Pangaro \\
\hline 103 & & Erythrinavariegata L. & Pangaro \\
\hline 104 & & Indigoferacordifolia Heyne ex Roth & \\
\hline 105 & & Indigoferaglandulosa Roxb. & \\
\hline 106 & & Indigoferatinctoria L. & \\
\hline 107 & & Paracalyxscariosus (Roxb.) Ali & \\
\hline 108 & & Pterocarpusmarsupium Roxb., var. acuminata Prain & Biyo \\
\hline 109 & & Rhynchosiaminima (L.) DC. & \\
\hline 110 & & Rhynchosiarothii Benth. ex. Ait & \\
\hline 111 & & Sesbaniabispinosa (Jacq.) Wight & \\
\hline 112 & & Sesbaniagrandiflora (L.) Pers. & \\
\hline 113 & & $\begin{array}{l}\text { Sesbaniasesban (L.) Merrill.subsp.sesban var. bicolor (W. } \\
\text { \&A.) F. W. Adrews }\end{array}$ & \\
\hline 114 & & $\underline{\text { Smithiasensitiva Ait. var. flava (Dalz.) Cooke }}$ & \\
\hline 115 & & Tephrosiatinctoria (L.) Pers. & \\
\hline 116 & & Tephrosiavillosa (L.) Pers. & \\
\hline 117 & & Teramnuslabialis (L.f.) Spreng. & \\
\hline 118 & & Vignaradiata (L.) Wilizeck. & \\
\hline 119 & & Vignaradiata (L.) Wilizeck. var. sublobata (Roxb.) Verdc. & \\
\hline 120 & & Vignatrilobata (L.) Verdc. & \\
\hline 121 & & Vignavexillata (L.) A.Rich. & \\
\hline 122 & \multirow[t]{14}{*}{ Caesalpiniaceae } & Bauhiniapurpurea $\mathrm{L}$. & Kanchnar \\
\hline 123 & & Bauhiniaracemosa Lamk. & Ashitro \\
\hline 124 & & Bauhiniatomentosa L. & \\
\hline 125 & & Caesalpiniapulcherrima (L.) Sw. & Galtaro \\
\hline 126 & & Cassiafistula L. & Garmalo \\
\hline 127 & & Cassiamimosoides L. & \\
\hline 128 & & Cassiaoccidentalis L. & \\
\hline 129 & & Delonixregia (Boj. G. HKP) Raf. & Gulmohar \\
\hline 130 & & Peltophorum sp. (Vogel)Benth. & \\
\hline 131 & & Peltophorumpterocarpum (DC.) Baker ex Heyne & Tamrafali \\
\hline 132 & & Sennasiamea (Lamk.) Irwin \& Barnby & Kasid \\
\hline 133 & & Senna auriculata Roxb. & Aval \\
\hline 134 & & Sennatora (L.) Roxb. & Kuvadiyo \\
\hline 135 & & Tamarindusindica L. & Amli \\
\hline 136 & \multirow[t]{8}{*}{ Mimosaceae } & Leucaenaleucocephala (Lamk.) De Wit. & Subaval \\
\hline 137 & & Mimosapudica L. & Lajamani \\
\hline 138 & & Pithecellobiumdulce (Roxb.) Benth. & Goras-aamli \\
\hline 139 & & Prosopisjuliflora (Sw.) DC. & Gando-baval \\
\hline 140 & & Vachelliafarnesiana (L.) Wight \& Arn. & Aniyar \\
\hline 141 & & Vachellialeucophloea (Roxb.) Rashmi Sharma & \\
\hline 142 & & Vachellianilotica (L.) P. J. H. Hurter \& Mabb. & Baval \\
\hline 143 & & Vachelliapennata (L.) U.C. Bapat \& Rashmi Sharma & Kher-vel \\
\hline 144 & \multirow[t]{2}{*}{ Vahliaceae } & Vahliadigyna (Retz.) O.Ketz. & \\
\hline 145 & & Anogeissussericosa Brandis & Adrukh \\
\hline
\end{tabular}




\begin{tabular}{|c|c|c|c|}
\hline 146 & Asclepiadaceae & Calycopterisfloribunda Lamk. & \\
\hline 147 & Combretaceae & Terminaliacatappa L. & Badam \\
\hline 148 & \multirow[t]{2}{*}{ Myrtaceae } & Psidiumguajava $\mathrm{L}$. & Jamfal \\
\hline 149 & & Syzygiumcumini (L.) Skeels & Jambu \\
\hline 150 & \multirow[t]{5}{*}{ Lythraceae } & Ammanniabaccifera L. & \\
\hline 151 & & Lagerstroemiaspeciosa (L.) Pers. & \\
\hline 152 & & Lagerstroemialanceolata Wall. & \\
\hline 153 & & Rotaladensiflora (Roxb.) GRRS Koehne & \\
\hline 154 & & Woodfordiafruticosa (L.) Kurz. & \\
\hline 155 & Onagraceae & Ludwigiaperennis L. & \\
\hline 156 & Caricaceae & Caricapapaya L. & Papaya \\
\hline 157 & \multirow[t]{12}{*}{ Cucurbitaceae } & Citrulluscolocynthis (L.) Schrad. & \\
\hline 158 & & Citrulluslanatus (Thunb) Matsumara \& Nakai & \\
\hline 159 & & Cocciniagrandis (L.) Voigt & Tindola \\
\hline 160 & & Cucumis melo $\mathrm{L}$. & \\
\hline 161 & & $\overline{\text { Cucumissativus L. }}$ L. & Kakadi \\
\hline 162 & & Cucurbitamaxima Duch. ex. Lamk. & Kolu \\
\hline 163 & & Lagenaria siceraria (Molina) Standl. & Dudhi \\
\hline 164 & & Luffa cylindrica (L.) M.J. Roem. & Turiya \\
\hline 165 & & Momordicadioica Roxb. ex. Willd. & \\
\hline 166 & & Solenaheterophylla Lour. & \\
\hline 167 & & Trichosanthes cucumerina L. & Jangli parval \\
\hline 168 & & Trichosanthesdioica Roxb. & \\
\hline 169 & Begoniaceae & Begoniacrenata Dryand & \\
\hline 170 & \multirow[t]{4}{*}{ Molluginaceae } & Glinuslotoides L. & \\
\hline 171 & & Glinusoppositifolius (L.) A. DC. & \\
\hline 172 & & Mollugopentaphylla L. & \\
\hline 173 & & Trianthemaportulacastrum L. & \\
\hline 174 & \multirow[t]{3}{*}{ Umbelliferae } & Cuminumcyminum L. & Jira \\
\hline 175 & & Daucuscarota L. & Gajar \\
\hline 176 & & Foeniculumvulgare Miller & Variyali \\
\hline 177 & Alangiaceae & Alangiumsalviifolium (L.f.) Wangerin & Ankol \\
\hline 178 & \multirow[t]{12}{*}{ Rubiaceae } & Borreriaarticularis (L.f.) F. N. Will. & \\
\hline 179 & & Catunaregam spinosa (Thunb.) Tirveng. & \\
\hline 180 & & Gardeniaresinifera Roth. & \\
\hline 181 & & Ixorabrachiata Roxb. & Ixora \\
\hline 182 & & Ixoracoccinea L. & Ixora \\
\hline 183 & & Meynalaxiflora Robyns & \\
\hline 184 & & Mitragynaparvifolia (Roxb.) Korth. & Kalam \\
\hline 185 & & Morindacitrifolia L. & Aal \\
\hline 186 & & Morindatomentosa Heyne ex Roth & Aal \\
\hline 187 & & Oldenlandiacorymbosa L. & \\
\hline 188 & & Oldenlandiaherbacea (L.) Roxb. & \\
\hline 189 & & Xeromphisuliginosa (Retz.) Maheshwari & \\
\hline 190 & \multirow[t]{9}{*}{ Asteraceae } & Acanthospermumhispidum DC. & \\
\hline 191 & & Ageratumconyzoides L. & \\
\hline 192 & & Bidensbipinnata L. & \\
\hline 193 & & Blumeaeriantha DC. & \\
\hline 194 & & Blumealacera (Burm. f.) DC. & \\
\hline 195 & & Caesuliaaxillaria Roxb. & \\
\hline 196 & & Conyzastricta Willd. & \\
\hline 197 & & Cyathoclinepurpurea (D.Don.) O. Ktze. & \\
\hline 198 & & Echinopsechinatus Roxb. & Kanto-selio \\
\hline
\end{tabular}




\begin{tabular}{|c|c|c|c|}
\hline 199 & & Ecliptaprostrata (L.) L. & Bhrungraj \\
\hline 200 & & Elephantopusscaber L. & \\
\hline 201 & & Emiliasonchifolia (L.) DC. & \\
\hline 202 & & Gnaphaliumluteo-album L. & \\
\hline 203 & & Grangeamaderaspatana (L.) Poir. & \\
\hline 204 & & Launeaprocumbens (Roxb.) Ramayya \& Rajgopal & \\
\hline 205 & & Partheniumhysterophorus L. & Congres grass \\
\hline 206 & & Plucheatomentosa DC. & \\
\hline 207 & & Sphaeranthusindicus L. & Gorakh-mundi \\
\hline 208 & & Spilanthescalva DC. & Akalkarar \\
\hline 209 & & Synedrellanodiflora (L.) Gaertn. & \\
\hline 210 & & Tricholepisamplexicaulis $\mathrm{Cl}$. & \\
\hline 211 & & Tridax procumbens (L.) L. & \\
\hline 212 & & Vernoniacinerea (L.) Less. & \\
\hline 213 & Plumbaginaceae & Plumbagozeylanica L. & Chitrak \\
\hline 214 & \multirow[t]{4}{*}{ Sapotaceae } & Manilkarahexandra (Roxb.) Dub. & Khirni, Rayan \\
\hline 215 & & Manilkarazapota (L.) var. royen & Chiku \\
\hline 216 & & Mimusopselengi L. & Borsali \\
\hline 217 & & Diospyrosmontana Roxb. & Timbaru \\
\hline 218 & \multirow[t]{2}{*}{ Oleaceae } & Jasminumhirsutum L. & \\
\hline 219 & & Nyctanthersarbortristis L. & Parijatak \\
\hline 220 & \multirow[t]{2}{*}{ Salvadoraceae } & Salvadoraoleoides Decne & Pilu \\
\hline 221 & & Salvadorapersica L. & Pilodi \\
\hline 222 & \multirow[t]{3}{*}{ Apocynaceae } & Pergulariadiamea (Forssk.) Chiv. & $\begin{array}{l}\text { Utarani, Kurmuda, } \\
\text { Rankaral }\end{array}$ \\
\hline 223 & & Cascabela thevetia (L.) Lippold & Pili karen \\
\hline 224 & & Tylophoradalzellii Hook.f. & \\
\hline 225 & \multirow[t]{3}{*}{ Periplocaceae } & Cryptolepisdubia (Burm.f.) Almeida & $\begin{array}{l}\text { Kali-kauli, } \\
\text { Krishna-sariva }\end{array}$ \\
\hline 226 & & Hemidesmusindicus (L.) Schult. & $\begin{array}{l}\text { Kauli, Anantmul, } \\
\text { Uparsal }\end{array}$ \\
\hline 227 & & $\begin{array}{l}\text { Hemidesmusindicus (L.) Schult. var. pubescens (Wt. \& } \\
\text { Arn.) Hk.f. }\end{array}$ & Kaulicha-vel \\
\hline 228 & Asclepiadaceae & Calotropis gigantea (L.) Dryand. & Akado \\
\hline 229 & Amaranthaceae & Dregeavolubilis (L. f.) Bth. ex. Hk. f. & \\
\hline 230 & \multirow[t]{3}{*}{ Asclepiadaceae } & Pergulariadaemia (Forsk). & Amar-dudheli \\
\hline 231 & & $\underline{\text { Saricostemmaacidum (L.) Holm. }}$. & \\
\hline 232 & & Telosmapallida (Roxb.) Craib & \\
\hline 233 & \multirow[t]{3}{*}{ Gentianaceae } & Canscora diffusa (Vahl) R.Br. ex Roem. \& Schult. & \\
\hline 234 & & Enicostema axillare (Poir. ex Lam.) A.Raynal & \\
\hline 235 & & Exacumpedunculatum L. & \\
\hline 236 & Menyanthaceae & Nymphoidesparvifolium (Griseb.) O. Ktze. & \\
\hline 237 & Hydrophyllaceae & Hydroleazeylanica (L.) Vahl. & \\
\hline 238 & Ehretiaceae & Cordiadichotoma Frost. & Gunda \\
\hline 239 & \multirow{6}{*}{ Boraginaceae } & Cordia sinensis Lam. & \\
\hline 240 & & Ehretiaaspera Roxb. & \\
\hline 241 & & Coldeniaprocumbens L. & \\
\hline 242 & & Heliotropiumindicum L. & Hathisundi \\
\hline 243 & & Heliotropiumpaniculatum R. Br. & \\
\hline 244 & & Trichodesma indicum (L.) R. Br. & \\
\hline 245 & \multirow[t]{4}{*}{ Convolvulaceae } & Argyreiasericea Dalz. & \\
\hline 246 & & Evolvulusalsinoides (L.) L. & Sankhpushpi \\
\hline 247 & & Evolvulusnummularins (L.) L. & \\
\hline 248 & & Ipomeaaquatica Forsk. & \\
\hline
\end{tabular}




\begin{tabular}{|c|c|c|c|}
\hline 249 & & Ipomeabatatas (L.) Lam. & Sakariya \\
\hline 250 & & Ipomeacairica (L.) Sw. & \\
\hline 251 & & Ipomoeaeriocapa R. Br. & \\
\hline 252 & & Ipomoeafistulosa Mart. ex Choisy & \\
\hline 253 & & Ipomoeahederifolia L. & \\
\hline 254 & & Ipomoeaindica (Burm.) Mem. & \\
\hline 255 & & Ipomoeanil (L.) Roth. & \\
\hline 256 & & Ipomoeaobscura (L.) Ker-Gawl. & \\
\hline 257 & & Ipomeapes-tigridis L. & \\
\hline 258 & & Ipomoea marginata (Desr.) Verdc. & \\
\hline 259 & & Ipomoeatriloba L. & \\
\hline 260 & & Merremiahederacea (Burm.f.) Hall. f. & \\
\hline 261 & & Merremia tridentata (L.) Hall. f. & \\
\hline 262 & & Merremiavitifolia (Burm. f.) Hall. f. & \\
\hline 263 & & Operculina turpethum (L.) Silva & \\
\hline 264 & & Riveahypocrateriformis. Choisy & \\
\hline 265 & Cuscutaceae & Cuscutachinensis Lam. & Amarvel \\
\hline 266 & Solanaceae & Capsicumannuum L. & Marcha \\
\hline 267 & & Physalisminima L. & \\
\hline 268 & & Solanumnigrum L. & \\
\hline 269 & & Withaniasomnifera (L.) Dunal & \\
\hline 270 & \multirow[t]{9}{*}{ Scrophulariaceae } & Bacopa monnieri (L.) Wettst. & Bam, Jalnaveri \\
\hline 271 & & Lindenbergiaindica (L.) O. Ktze. & $\begin{array}{l}\text { Pirsadedi, } \\
\text { Zamarval, } \\
\text { Patharchati }\end{array}$ \\
\hline 272 & & Linderniaantipoda (L.) Alst. & \\
\hline 273 & & Linderniaciliata (Colsm.) Pennell & \\
\hline 274 & & Linderniaparviflora (Roxb.) Haines & \\
\hline 275 & & Scopariadulcis L. & \\
\hline 276 & & Sopubiadelphinifolia (L.) G. Don. & \\
\hline 277 & & Verbascumchinese (L.) Santapau & Kalhar, Kolhala \\
\hline 278 & & Veronicaanagallis- aquatica L. & \\
\hline 279 & Orobancaceae & $\underline{\text { Strigaangustifolia (D. Don) Saldhana }}$ & $\begin{array}{l}\text { Dholo agio, } \\
\text { Kunvario agio }\end{array}$ \\
\hline 280 & \multirow{5}{*}{ Bignoniaceae } & Dolichandronespathacea (L.f.) K. Schum. & Medsingi \\
\hline 281 & & Millingtoniahortensis L. & Deshi Buch \\
\hline 282 & & Oroxylumindicum (L.) Vent. & Tetu \\
\hline 283 & & Tabebuiapentaphylla (L.) Hemsl. & \\
\hline 284 & & Tecoma stans (L.) Juss. ex Kunth & \\
\hline 285 & \multirow[t]{2}{*}{ Pedaliaceae } & Pedaliummurex L. & Ubhu Gokhru \\
\hline 286 & & $\underline{\text { Sesamumindicum } \mathrm{L} .}$ & Tal \\
\hline 287 & Martyniaceae & Martyniaannua L. & Vinchhudo \\
\hline 288 & \multirow[t]{11}{*}{ Acanthaceae } & Adhatodavasica (L.) Nees. & Ardusi \\
\hline 289 & & Blepharis maderaspatensis (L.) B.Heyne ex Roth. & $\begin{array}{l}\text { Untigan, Utanjan } \\
\text { chokd }\end{array}$ \\
\hline 290 & & Elytrariaacaulis (L.f.) Lindau & \\
\hline 291 & & Hygrophilaauriculata (Schum.) Heine & \\
\hline 292 & & Justiciagendarussa Burm. f. & \\
\hline 293 & & Justicia japonica Thunb. & \\
\hline 294 & & Lepidagathiscristata Willd. & \\
\hline 295 & & Lepidagathistrinervia Wall. & Harancharo, Paniru \\
\hline 296 & & Neuracanthussphaerostachyus (Nees.) Dalz. & Ganthera \\
\hline 297 & & Ruelliatuberosa L. & Fatkai \\
\hline 298 & & Rungiapectinata (L.) Nees & Khadsello \\
\hline
\end{tabular}




\begin{tabular}{|c|c|c|c|}
\hline 299 & & Rungiarepens. (L.) Nees & \\
\hline 300 & & Thunbergiaerecta (Bth.) T. Anders. & \\
\hline 301 & & Thunbergiagrandiflora (Roxb ex Rottl.) Roxb. & \\
\hline 302 & \multirow[t]{7}{*}{ Verbenaceae } & Clerodendruminerme (L.) Gaertn. & \\
\hline 303 & & Clerodendrumserratum (L.) Moon & Bharungi \\
\hline 304 & & Gmelinaarborea Roxb. & Sivan \\
\hline 305 & & Gmelinaasiatica L. & \\
\hline 306 & & Gmelina phillippensis Cham. & \\
\hline 307 & & Lantanacamara L. var. aculeata (L.) Mold. & Lantana \\
\hline 308 & & Vitexnegundo L. & Nagod, Nagud \\
\hline 309 & \multirow[t]{5}{*}{ Lamiaceae } & Hyptissuaveolens (L.) Poit. & \\
\hline 310 & & Leucas aspera (Willd.) Link. & Kubi \\
\hline 311 & & Leucas biflora (Vahl) Sm. & \\
\hline 312 & & Plectranthusmollis (Ait.) Spreng. & \\
\hline 313 & & Pogostemonparviflora Bth. & \\
\hline 314 & \multirow[t]{4}{*}{ Nyctaginaceae } & Boerhaviadiffusa L. & Satodi \\
\hline 315 & & BoerhaviaChinensis (L.) Rottb. & \\
\hline 316 & & Mirabilisjalapa L. & Gulbas \\
\hline 317 & & Pisoniamitis L. & Velati, Salet \\
\hline 318 & \multirow[t]{6}{*}{ Amaranthaceae } & AchyranthesasperaL. & Anghedi \\
\hline 319 & & Aervalanata (L.) Juss.ex Sch. & Kapuri madhuri \\
\hline 320 & & Amaranthus spinosus L. & \\
\hline 321 & & Amaranthusviridis L. & \\
\hline 322 & & Gomphrenacelosioides Mart. & \\
\hline 323 & & Pupalialappacea (L.) Juss. & \\
\hline 324 & Basellaceae & Basellarubra L. & Poi \\
\hline 325 & \multirow[t]{2}{*}{ Polygonaceae } & Antigononleptopus Hook. \& Arn. & Ice cream creeper \\
\hline 326 & & Persicariaglabra (Willd.) Gomez. & \\
\hline 327 & \multirow[t]{2}{*}{ Loranthaceae } & Dendrophthoefalcata (L. f.) Ettingsh. & Vando \\
\hline 328 & & Viscumarticulatum Burm.f. & \\
\hline 329 & Santalaceae & Santalumalbum L. & Chandan \\
\hline 330 & \multirow[t]{18}{*}{ Euphorbiaceae } & Acalyphaciliata Forsk. & Dadari \\
\hline 331 & & Acalyphaindica L. & Dadari \\
\hline 332 & & Brideliaspinosa Willd. & Asan \\
\hline 333 & & Brideliasquamosa (Lam.) Gehrm. & Asan \\
\hline 334 & & Chamaesycehirta (L.) Millsp. & \\
\hline 335 & & Chamaecycethymifolia (L.) Millsp. & \\
\hline 336 & & Chrozophorarottleri (Geis.) Juss. & Okharad \\
\hline 337 & & Euphorbiaantiquorum L. & Thor \\
\hline 338 & & Euphorbianeriifolia L. & Thor \\
\hline 339 & & Jatrophagossypiifolia L. & \\
\hline 340 & & Kirganelliareticulata (Poir) Bail. & Pichrun \\
\hline 341 & & Mallotusphilippensis (Lamk.) Muell.-Arg. & Kapilo \\
\hline 342 & & Pedilanthustithymaloides (L.) Poit. & Vilayati-kharsan \\
\hline 343 & & Phyllanthusemblica L. & Aavla \\
\hline 344 & & Phyllanthuserecta (Medic.) Almeida & Bhonya amli \\
\hline 345 & & Phyllanthusmaderaspatensis L. & Bakarato \\
\hline 346 & & Ricinuscommunis L. & \\
\hline 347 & & Securinegaleucopyros (Willd.) Muell.-Arg. in DC. & Chhini, Shenvi \\
\hline 348 & \multirow[t]{2}{*}{ Ulmaceae } & Holopteleaintegrifolia (Roxb.) Planch. & Kanjo, Papda \\
\hline 349 & & Tremaorientalis (L.) Blume & Gol \\
\hline 350 & \multirow[t]{2}{*}{ Moraceae } & Artocarpusheterophyllus Lamk. & Phanas, Jack-fruit \\
\hline 351 & & Ficusbenghalensis L. & Vad \\
\hline
\end{tabular}




\begin{tabular}{|c|c|c|c|}
\hline 352 & & Ficuscarica L. & Anjir \\
\hline 353 & & Ficushispida L.f. & Dhedh Umbaro \\
\hline 354 & & Ficusracemosa L. & Umbaro \\
\hline 355 & & Ficusreligiosa L. & Pipal \\
\hline 356 & & Ficusvirens Dryand & Payar \\
\hline 357 & & Morusalba L. & Shetur \\
\hline 358 & & Streblusasper Lour. & Harero \\
\hline 359 & Costaceae & Costusspeciosus (Koen. ex Retz.) Smith & Pavuta \\
\hline 360 & Amaryllidaceae & Crinumasiaticum L. & \\
\hline 361 & Taccaceae & Taccaleontopetaloides (L.) Kuntze & \\
\hline 362 & Liliaceae & Gloriosasuperba L. & \\
\hline 363 & Pontederiaceae & Eichhorniacrassipes (Mart.) Solms. & Kanphutti \\
\hline 364 & \multirow[t]{6}{*}{ Commelinaceae } & Amischophaceluscucullata (Roth) Rolla Rao \& Kammathy & \\
\hline 365 & & Commelinabenghalensis $\mathrm{L}$. & \\
\hline 366 & & Commelinadiffusa Burm.f. & \\
\hline 367 & & $\overline{\text { Commelinaerecta L. }}$. & \\
\hline 368 & & Cyanotiscristata (L.) D. Don & \\
\hline 369 & & Murdannianudiflora (L.) Brenan & \\
\hline 370 & \multirow[t]{2}{*}{ Arecaceae } & Arecacatechu L. & Sopari \\
\hline 371 & & Borassusflabelifer L. & $\mathrm{Tad}$ \\
\hline 372 & Typhaceae & Typhadomingensis Pers. & \\
\hline 373 & Araceae & Amorphophalluscommutatus Engler & \\
\hline 374 & Lemnaceae & Lemnagibba L. & \\
\hline 375 & Lemnaceae & Wolffiaarrhiza (L.) Wimmer & \\
\hline 376 & Potamogetonaceae & Potamogetoncrispus L. & \\
\hline 377 & \multirow[t]{15}{*}{ Cyperaceae } & Cyperusalternifolius L. & Umbrella plant \\
\hline 378 & & Cyperusbulbosus Vahl & \\
\hline 379 & & Cyperuscompressus L. & \\
\hline 380 & & Cyperuscorymbosus Rottb. & \\
\hline 381 & & Cyperusdifformis L. & \\
\hline 382 & & Cyperusesculentus L. & \\
\hline 383 & & Cyperusexaltatus Retz. & \\
\hline 384 & & Cyperusiria L. & \\
\hline 385 & & Cyperusrotundus L. & Chido \\
\hline 386 & & Eleocharisatropurpurea (Retz.) Presl. & \\
\hline 387 & & Fimbristylisbis-umbellata (Forsk.) Bub. & \\
\hline 388 & & Fimbristylisdichotoma (L.) Vahl, Cooke & \\
\hline 389 & & Fuirenaciliaris (L.) Roxb. & \\
\hline 390 & & Schoenoplectusarticulatus (L.) Palla & \\
\hline 391 & & Apludamutica L. & Harantodi, Godval \\
\hline 392 & Poaceae & Aristidaadscensionis L. & \\
\hline 393 & & Bambusaarundinacea (Retz.) Willd. & \\
\hline 394 & & Brachiariaramosa (L.) Stapf & \\
\hline 395 & & Chlorisbarbata Sw. & Mindadiu \\
\hline 396 & & Chrysopogonfulvus (Spreng.) Chiov. & Draf, Kharalu \\
\hline 397 & & Coixlachryma-jobi L. & Kahudo, Kasai \\
\hline 398 & & Cymbopogoncitratus (DC.) Stapf & Lili chaha \\
\hline 399 & & Cynodondactylon (L.) Pers. & Darbh \\
\hline 400 & & Dactylocteniumaegypticum (L.) Willd. & \\
\hline 401 & & Dendrocalamusstrictus (Roxb.) Nees & Narvans \\
\hline 402 & & Desmostachyabipinnata (L.) Stapf in Dyer & Manga, Darbha \\
\hline 403 & & Dichanthiumannulatum (Forssk.) Stapf & \\
\hline 404 & & Digitariaciliaris (Retz.) Koeler & \\
\hline
\end{tabular}




\begin{tabular}{|l|l|l|}
\hline 405 & Echinochloacolona (L.) Link & Samo \\
\hline 406 & Eleusinecoracana (L.) Gaertn. & Nagli \\
\hline 407 & Eleusineindica (L.) Gaertn. & Ukdo \\
\hline 408 & Eragrostistenella (L.) P. Beauv. ex Roem. \& Schult. & \\
\hline 409 & Eragrostisuniloides (Retz.) Nees ex Steud. & \\
\hline 410 & Eremopogonfoveolatus (Delile) Stapf & \\
\hline 412 & Heteropogoncontortus (L.) P. Beauv. ex Roem. \& Schult. & \\
\hline 413 & Ischaemumrugosum Salib. & \\
\hline 414 & Oplismenuscompositus (L.) P. Beauv. & Bhat, Chokha \\
\hline 415 & Oryzasativa L. & Jinko-samo \\
\hline 416 & Paspalidiumflavidum (Retz.) A. Camus & Bajra \\
\hline 417 & $\underline{\text { Pennisetumglaucum (L.) R.Br. }}$ & Sherdi \\
\hline 418 & Saccharumofficinarum L. & \\
\hline 419 & Setariaglauca (L.) Beauv. & Baru \\
\hline 420 & Sorghumhalepense (L.) Pers. & Ghahun \\
\hline
\end{tabular}

\section{Conclusion:-}

In the present study 420 species and 330 genera of flowering plants belonging to 91 families which provide basics for survival for others animal including birds, reptiles, amphibians and insects. Many wetlands are vital bird's habitats and bird's usage them as migratory resorts for breeding, nesting and rearing young once. Some water birds like Grebes have adapted to wetlands to such range to survive as an individual species depends on the accessibility of food and shelter in certain types of wetlands within their geographic ranges.

\section{References:-}

1. Ali S and Ripley SD (1987) Compact Handbook of the Birds of India and Pakistan. Oxford University Press, Bombay.

2. Blackman JG, Gardiner SJ, Morgan MG (1995) Framework for biogeographic inventory, assessment, planning and management of wetland systems: The Queensland approach. Wetland research in the Wet-Dry tropics of Australia.

3. Brown M and Dinsmore JJ (1986) Implications of marsh size and isolation for marsh bird management. The Journal of Wildlife Management, 1:392-7.

4. Bryce SA, Hughes RM, Kaufmann PR. Development of a bird integrity index: using bird assemblages as indicators of riparian condition. Environmental management. 2002 Aug 1;30(2):294-310.

5. Cherry JA (2012) Ecology of wetland ecosystems: water, substrate, and life. Nature Education Knowledge, 3(10): 16.

6. Cooke T (1967) The Flora of the Presidency of Bombay. Volume I, II and III. Botanical Survey of India, Calcutta.

7. Finlayson CM (1995) Wetland research in the wet-dry tropics of Australia. In Workshop, Jabiru NT, 22-24.

8. Finlayson CM (1999.) Information needs for wetland management. Max Finlayson and Abbie Speirs.

9. Finlayson CM, Hall R, Bayliss B (1998) Regional review of wetlands management issues: wet-dry tropics of northern Australia. Land and Water Resources Research and Development Corporation.

10. Gonzalves EA and Joshi DB (1985) The seasonal sucession of the algae in a tank of Bandra. Journal of Bombay Natural History Society. 46: 154-176.

11. Gopal B (2005) Biodiversity in Freshwater Ecosystems Including Wetlands, Biodiversity and Conservation in India, A Status Report.

12. Gosselink JG and Turner RE (1978) Role of hydrology in freshwater wetland ecosystems. Freshwater wetlands.

13. Groombridge B and Jenkins M (1998) Freshwater biodiversity: a preliminary global assessment.Mitsch WJ and Gosselink JG (2000) The value of wetlands: importance of scale and landscape setting. Ecological Economics, 35(200): 25-33.

14. Nirmal Kumar JI, Kumar RN, Bhatt I (2005) Study of Cultural Eutrophication in Relation to Plant Diversity of Wetland: Ratheshwar in Central Gujarat. Aquatic Biodiversity in India: The Present Scenario, 243.

15. Parikh P, Unadkat K and Nagar P (2015) Study of aquatic weeds in two ponds of Vadodara, Gujarat. IJAPRR (International Peer Reviewed Refereed Journal), 2(1);1-7. 
16. Prasad SN, Ramachandra TV, Ahalya N, Sengupta T, Kumar A, Tiwari AK, Vijayan VS and Vijayan L (2002) Conservation of wetlands of India-a review. Tropical Ecology, 43(1):173-86.

17. Shah GL (1978) The flora of Gujarat State. Vol-I and II. Sardar Patel University Press, Vallabh Vidhyanagar, Anand, India.

18. Singh DH (2003) Marine Protected Areas of India: Status of Coastal Wetland Conservation. Gujarat Ecological Education and Research Foundation, Gandhinagar.

19. Solanki HA (2001) Study on pollution of soils and water reservoirs near industrial areas of Baroda. Ph.D Thesis submitted to Bhavnagar University, Bhavnagar.

20. Soni HB, Dabhi M and Thomas S (2013) Surface water quality assessment and conservation measures of two pond ecosystems of Central Gujarat. International Research Journal of Chemistry, 3(3):69-81.

21. Verboom J, Foppen R, Chardon P, Opdam P and Luttikhuizen P (2001) Introducing the key patch approach for habitat networks with persistent populations: an example for marshland birds. Biological conservation, 100(1):89-101. 